\title{
NUMERICAL ASPECTS OF THE RADIAL INTEGRATION METHOD IN BOUNDARY ELEMENT FORMULATION FOR STABILITY ANALYSIS OF THIN PERFORATED PLATES OF LAMINATED COMPOSITES
}

\author{
P. C. M. Doval ${ }^{1}$, E. L. Albuquerque ${ }^{2}$, P. Sollero ${ }^{3}$ \\ ${ }^{1}$ Department of Mechanical and Material, Federal Institute of Maranhão (doval@ fem.unicamp.br) \\ ${ }^{2}$ Faculty of Technology, University of Brasília, Campus Universitário Darcy Ribeiro \\ ${ }^{3}$ Department of Computational Mechanics, State University of Campinas
}

\begin{abstract}
The radial integration method is a suitable technique to transform domain integrals into boundary integrals. It is quite appropriated for anisotropic materials because it is a pure numerical technique that does not require the computation of approximation functions as in dual reciprocity boundary element method. However, a special attention must be paid on the numerical integration because it has strong influence on the accuracy and computational cost of the method. This paper presents an analysis of performance of the radial integration method, considering accuracy and computational cost, when it is used in stability analysis of thin perforated plates of laminated composite plates by the boundary element formulation. The accuracy of the proposed formulation is assessed by comparison with results from the literature.
\end{abstract}

Keywords: Stability of structures, linear buckling, laminated composite plates, radial integration method, boundary element method.

\section{INTRODUCTION}

This paper presents an analysis of performance of the radial integration method, considering accuracy and computational cost, when it is used in stability analysis of perforated thin plates of laminated composite by the boundary element formulation. The classical plate bending and the plane elasticity formulations [1] are used and the domain integrals due to non-uniform body forces are transformed into boundary integrals using the radial integration method. The presented formulation does not require neither domain discretization nor computation of particular solutions.

The formulation of the boundary element method for anisotropic plane elasticity was developed by [2] to problems of fracture mechanics and elasto-static and extended to other problems in the research of [3]. The formulation of boundary elements for the classical theory of anisotropic plates was developed by $[4,5]$, and extended to other problems in the work of 
[6]. The formulation for stability analysis of thin perforated plates of laminated composite was developed by [7]. The last work has used the radial integration method for the transformation of domain integrals that remain in the formulation into boundary integrals. The computational cost of this formulation was high however, especially for anisotropic formulation.

The main contribution of this paper is to examine the sensitivity of the radial integration method in relation to the number of integration points for the formulation of thin perforated plates of laminated composite. As quoted in the literature, in [8] and [9], the radial integration method demand few integration points to obtain a solution close to the analytical solution. In these two studies, good results are obtained with ten integration points. However, this was not carried out for stability analysis of thin perforated plates of laminated composite. The main focus of this paper is to assess the number of integration points that are needed to obtain results with good accuracy.

\section{GOVERNING EQUATIONS}

Basically, the classic problem of buckling is a geometrically nonlinear problem described by a set of three differential equations which can be uncoupled and linearized in the case of elastic critical loads. In the absence of body forces, equations that describe the buckling of plates are given by:

$$
N_{i j, j}=0,
$$

$$
D_{11} u_{3,1111}+4 D_{16} u_{3,1112}+2\left(D_{12}+D_{66}\right) u_{3,1122}+4 D_{26} u_{3,1222}+D_{22} u_{3,2222}=N_{i j} u_{3, i j}
$$

where $i, j, k=1,2 ; u_{k}$ is the displacement in directions $x_{1}$ and $x_{2}, u_{3}$ stands for the displacement in the normal direction of the plate surface; $N_{i j}$ are the in-plane stress components, $D_{11}$, $D_{22}, D_{66}, D_{12}, D_{16}$, and $D_{26}$ are the anisotropic thin plate stiffness constants.

\subsection{Boundary integral equations}

The determination of in-plane stress resultants in the domain is the first step in the solution of plate buckling. The in-plane boundary integral equation for displacements, obtained by applying the reciprocity and Green theorems in equation (1), is given by [10]:

$$
c_{i j} u_{j}(Q)+\int_{\Gamma} t_{i k}^{*}(Q, P) u_{k}(P) d \Gamma(P)=\int_{\Gamma} u_{i k}^{*}(Q, P) t_{k}(P) d \Gamma(P),
$$

where $t_{i}=N_{i j} n_{j}$ is the traction in the boundary of the plate in the plane $x_{1}-x_{2}$, and $n_{j}$ is the normal at the boundary point; $P$ is the field point; $Q$ is the source point; and asterisks denote fundamental solutions. The anisotropic plane elasticity fundamental solutions can be found, for example, in [11]. The constant $c_{i j}$ is introduced in order to take into account the possibility that the point $Q$ can be placed in the domain, on the boundary, or outside the domain.

The in-plane stress resultants at a point $Q \in \Omega$ are written as: 


$$
c_{i k} N_{k j}(Q)+\int_{\Gamma} S_{i k j}^{*}(Q, P) u_{k}(P) d \Gamma(P)=\int_{\Gamma} D_{i j k}^{*}(Q, P) t_{k}(P) d \Gamma(P),
$$

where $D_{i k j}$ and $S_{i k j}$ are linear combinations of the plane-elasticity fundamental solutions. Due to stress concentrations in the geometry, stress resultants are non-uniform over the domain.

The plate buckling equations are derived from the plate bending equations. Critical load factors are introduced into the equations as multiplication factors of body forces or transverse loads. Critical buckling loads are loads at which plates suddenly undergo considerable deflections in the transverse direction due to loads applied in the plane of the plate. The relation between the applied load and critical loads are given by the critical load factor $\lambda$ by the following equation:

$$
N_{i j}^{c}=\lambda N_{i j}
$$

where $N_{i j}^{c}$ are critical stress resultants that are obtained when critical loads are applied.

The integral equation for the plate buckling formulation, obtained by applying reciprocity and Green theorems at equation (2), is given by:

$$
\begin{aligned}
K u_{3}(Q) & +\int_{\Gamma}\left[V_{n}^{*}(Q, P) w(P)-m_{n}^{*}(Q, P) \frac{\partial w(P)}{\partial n}\right] d \Gamma(P)+\sum_{i=1}^{N_{c}} R_{c_{i}}^{*}(Q, P) u_{3_{c i}}(P) \\
= & \sum_{i=1}^{N_{c}} R_{c_{i}}(P) u_{3_{c i}}^{*}(Q, P)+\int_{\Gamma}\left[V_{n}(P) u_{3}^{*}(Q, P)-m_{n}(P) \frac{\partial u_{3}^{*}}{\partial n}(Q, P)\right] d \Gamma(P) \\
+ & \lambda\left[\int_{\Omega} u_{3} N_{i j} u_{3, i j}^{*} d \Omega+\int_{\Gamma}\left(t_{i} u_{3}^{*} u_{3, i}-t_{i} u_{3} u_{3, i}^{*}\right) d \Gamma\right]
\end{aligned}
$$

where $\frac{\partial()}{\partial n}$ is the derivative in the direction of the outward vector $\mathbf{n}$ that is normal to the boundary $\Gamma ; m_{n}$ e $V_{n}$ are, respectively, the normal bending moment and the Kirchhoff equivalent shear force on the boundary $\Gamma ; R_{c}$ is the thin-plate reaction of corners; $u_{3_{c i}}^{*}$ is the transverse displacement of corners; $\lambda$ is the critical load factor; the constant $K$ is introduced in order to take into account the possibility that the point $Q$ can be placed in the domain, on the boundary, or outside the domain. As in the previous equation, an asterisk denotes a fundamental solution. Fundamental solutions for anisotropic thin plates can be found, for example, in [5].

A second integral equation is necessary in order to obtain the thin plate buckling boundary element formulation. This equation is obtained by the derivative of equation (6) in respect to the normal direction at the source point $Q$. This equation is given by:

$$
\begin{gathered}
K \frac{\partial u_{3}}{\partial m}(Q)+\int_{\Gamma}\left[\frac{\partial V_{n}^{*}}{\partial m}(Q, P) w(P)-\frac{\partial M_{n}^{*}}{\partial m}(Q, P) \frac{\partial u_{3}(P)}{\partial n}\right] d \Gamma(P)+\sum_{i=1}^{N_{c}} \frac{\partial R_{c_{i}}^{*}}{\partial m}(Q, P) u_{3_{c i}}(P) \\
=\sum_{i=1}^{N_{c}} R_{c_{i}}(P) \frac{\partial u_{3_{c i}}^{*}}{\partial m}(Q, P)+\int_{\Gamma}\left[V_{n}(P) \frac{\partial u_{3}^{*}(Q, P)}{\partial m}-m_{n}(P) \frac{\partial^{2} u_{3}^{*}}{\partial n \partial m}(Q, P)\right] d \Gamma(P) \\
+\lambda\left[\int_{\Omega} u_{3} N_{i j} \frac{\partial u_{3, i j}^{*}}{\partial m} d \Omega+\int_{\Gamma}\left(t_{i} u_{3}^{*} \frac{\partial u_{3, i}}{\partial m}-t_{i} u_{3} \frac{\partial u_{3, i}^{*}}{\partial m}\right) d \Gamma\right]
\end{gathered}
$$


where $\frac{\partial()}{\partial m}$ is the derivative with respect to the direction of the outward vector $\mathbf{m}$ that is normal to the boundary $\Gamma$ at the source point $Q$.

As it can be seen in equations (6) and (7), domain integrals arise in the formulation owing to the contribution of in-plane stresses to the out of plane direction. In order to transform these integrals into boundary integrals, consider that a body force $b$ is approximated over the domain as a sum of $M$ products between approximation functions $f_{m}$ and unknown coefficients $\gamma_{m}$, that is:

$$
b(P) \cong \sum_{m=1}^{M} \gamma_{m} f_{m} .
$$

Equation (8) can be written in a matrix form, considering all boundary and domain source points, as:

$$
\mathbf{b}=\mathbf{F} \gamma
$$

Thus, $\gamma$ can be computed as:

$$
\gamma=\mathbf{F}^{-1} \mathbf{b}
$$

Body forces of integral equations (6) and (7) depend on displacements. So, using equation (10) and following the procedure presented by [12], domain integrals that come from these body forces can be transformed into boundary integrals.

As it can be seen in equations (6) and (7), the body force that generates domain integrals is given by:

$$
b=N_{i j} u_{3}
$$

So, it's need to compute $N_{i j}$ in each integration points. However, we have only the values of $N_{i j}$ at nodes and internal points. Values of $N_{i j}$ in integration points is computed by:

$$
N_{i}\left(x_{1}, x_{2}\right)=f(r) F^{-1} N_{i j} .
$$

The implementaion of the buckling formulation is quite similar to shells. Details on the implementation of the radial integration method for shells are given by [13].

\subsection{Approximation function}

The approximation function used in this work is:

$$
f_{m}=r^{2} \log (r),
$$

The function (13) is known as thin plate splines, where $r$ is the distance between the centre of the radial basis function and integration points. It has been shown in some works from literature that this approximation function can give excellent results for many different formulations (see [14] and [15]). 


\subsection{Eigenvalue problem}

After the discretization of equations (6) and (7) into boundary elements and collocation of the source points in all boundary nodes, a linear system is generated. It is worth notice that the only loads considered in the linear buckling equations are that related to the in-plane stress $N_{i j}$ and tractions $t_{i}$ that are multiplied by the critical load factor $\lambda$. Furthermore, all the known values of $w, \partial w / \partial n, M_{n}, V_{n}, w_{c i}, R_{c i}$ (boundary conditions) are set to zero. Dividing the boundary into $\Gamma_{1}$ e $\Gamma_{2}$ (Figure 4 ), this linear system can be written as:

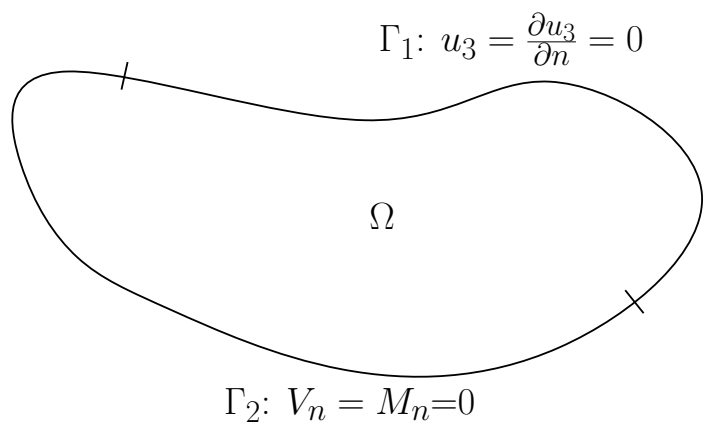

Figure 1. Domain with constrained and free degrees of freedom.

$$
\left[\begin{array}{ll}
H_{11} & H_{12} \\
H_{21} & H_{22}
\end{array}\right]\left\{\begin{array}{l}
w_{1} \\
w_{2}
\end{array}\right\}-\left[\begin{array}{ll}
G_{11} & G_{12} \\
G_{21} & G_{22}
\end{array}\right]\left\{\begin{array}{l}
V_{1} \\
V_{2}
\end{array}\right\}=\lambda\left[\begin{array}{ll}
M_{11} & M_{12} \\
M_{21} & M_{22}
\end{array}\right]\left\{\begin{array}{l}
w_{1} \\
w_{2}
\end{array}\right\}
$$

where $\Gamma_{1}$ stands for the part of the boundary where displacements and rotations are zero and $\Gamma_{2}$ stands for the part of the boundary where bending moment and tractions are zero. Indices 1 and 2 stand for boundaries $\Gamma_{1}$ and $\Gamma_{2}$, respectively. Matrices $\mathbf{H}, \mathbf{G}$, and $\mathbf{M}$ are influence matrices of the boundary element method due to integral terms of equations (6) and (7).

As $\mathbf{w}_{\mathbf{1}}=\mathbf{0}$ and $\mathbf{V}_{\mathbf{2}}=\mathbf{0}$, equation (14) can be written as:

$$
\begin{aligned}
& \mathbf{H}_{12} \mathbf{w}_{2}-\mathbf{G}_{11} \mathbf{V}_{1}=\lambda \mathrm{M}_{12} \mathbf{w}_{2} \\
& \mathbf{H}_{22} \mathbf{w}_{2}-\mathbf{G}_{21} \mathbf{V}_{1}=\lambda \mathrm{M}_{22} \mathbf{w}_{2}
\end{aligned}
$$

or,

$$
\hat{\mathbf{H}} \mathrm{w}_{2}=\lambda \hat{\mathbf{M}} \mathrm{w}_{2} \text {, }
$$

where, $\hat{\mathbf{H}}$ e $\hat{\mathbf{M}}$, are given by:

$$
\begin{aligned}
\hat{\mathbf{H}} & =\mathrm{H}_{22}-\mathrm{G}_{21} \mathrm{G}_{11}^{-1} \mathbf{H}_{12} \\
\hat{\mathbf{M}} & =\mathbf{M}_{22}-\mathrm{G}_{21} \mathrm{G}_{11}^{-1} \mathbf{M}_{12}
\end{aligned}
$$

The matrix equation (16) can be rewritten as an eigen vector problem

$$
\mathbf{A} \mathbf{w}_{\mathbf{2}}=\frac{1}{\lambda} \mathbf{w}_{\mathbf{2}},
$$


where,

$$
\mathbf{A}=\hat{\mathbf{H}}^{-1} \hat{\mathbf{M}}
$$

Provided that $\mathbf{A}$ is non-symmetric, eigenvalues and eigenvectors of equation (18) can be found using standard numerical procedures for non symmetric matrices.

\section{NUMERICAL RESULTS}

In order to assess the accuracy of the proposed formulation, in this work it is considered a square thin plate of laminated composite with a square hole, under uniformly uniaxial compression and the critical load parameter is computed considering all edges simplysupported, shown in figures 2 and 3. The geometry and material properties of the plate are: ratio between length $a$ and thickness $h$ of the square plate is $a / h=100$; ratio between the edge length of the plate and the edge length of the hole is $a / b=5$; elastic moduli $E_{1}=181$ $G P a$ and $E_{2}=10.3 G P a$ and Poisson ratio $\nu_{12}=0.28$, and shear modulus $G_{12}=7.17 G P a$.

The numerical results are presented in terms of the dimensionless critical load parameter $K_{c r}$ which is given by:

$$
K_{c r}=\frac{N_{c r} a^{2}}{D_{22}}
$$

where, $N_{c r}$ is the critical load and $a$ is the edge length of the square plate.

It's used three meshes with quadratic discontinuous boundary elements: mesh 1 has 12 elements of equal length at the external boundary and 12 elements of equal length at the hole and 48 uniformly distributed internal points; mesh 2 has 20 elements of equal length at the external boundary and 12 elements of equal length at the hole and 48 uniformly distributed internal points; mesh 3 has 28 elements of equal length at the external boundary and 12 elements of equal length at the hole and 48 uniformly distributed internal points.

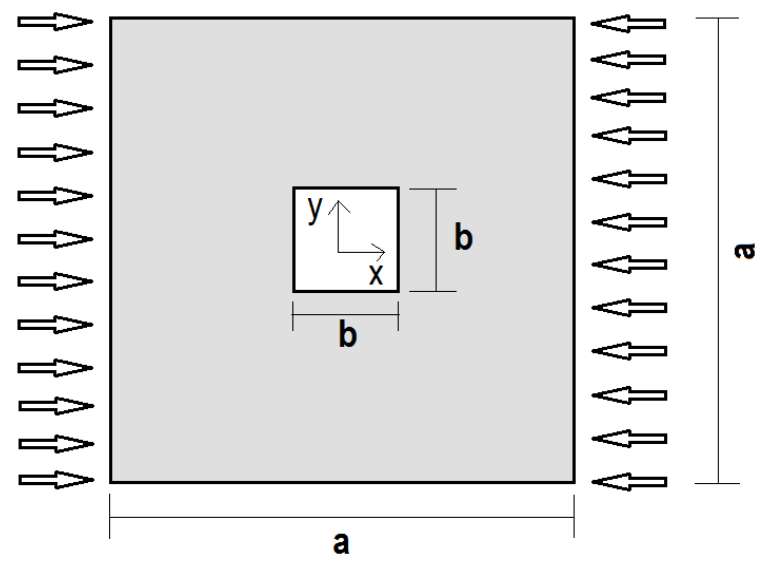

Figure 2. Geometric configuration

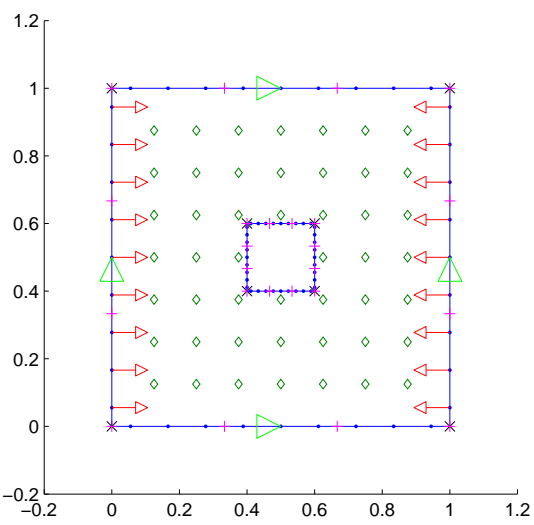

Figure 3. Boundary element model (mesh 1)

The results for a square composite laminate plate with square hole are shown in Table 1 using the radial integration method in boundary element formulation. The solution presented by [7] to this problem is $K_{c r}=112.30$. 
Table 1. Critical load parameter $K_{c r}$ for a perforated square plate of composite laminated

\begin{tabular}{|c|c|c|c|c|c|c|c|c|}
\hline \multirow{2}{*}{ Mesh } & \multicolumn{8}{|c|}{ Number of integration points } \\
\cline { 2 - 9 } & 4 & 8 & 12 & 16 & 20 & 24 & 26 & 30 \\
\hline 1 & 25.89 & 116.85 & 144.66 & 134.59 & 125.11 & 118.25 & 118.25 & 118.25 \\
\hline 2 & 61.43 & 100.28 & 113.58 & 110.22 & 114.66 & 114.66 & 114.66 & 114.66 \\
\hline 3 & 67.88 & 96.08 & 101.55 & 107.18 & 112.02 & 112.20 & 112.20 & 112.20 \\
\hline
\end{tabular}

\section{DISCUSSION OF THE RESULTS}

As can be seen in figure 4, the resultas for $K \mathrm{cr}$ converge to the solution presented by [7] with mesh refinement. Comparing the results obtained in Table 1, column corresponding to 24 integration points, for all three meshes, we notice that differences are always smaller than $6 \%$.

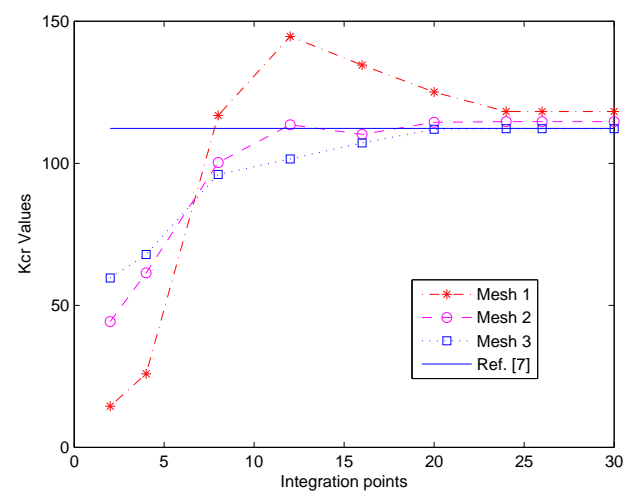

Figure 4. Comparison of the values $K c r$ and the number of integration points

\section{CONCLUSIONS}

This paper presented an analysis of performance of the radial integration method, considering accuracy and computational cost, when it is used in a boundary element formulation for the instability analysis of composite laminate plates with non-uniform stress field. Domain integrals are transformed into boundary integrals by the radial integration method.

This result makes this method very suitable for the treatment of these types of problems, since the computational cost is not high and the fact that we do not need to calculate particular solutions makes the radial integration method advantageous, because of the easy implementation, when compared with the dual reciprocity boundary element method.

It was shown that very good results can be obtained with 24 integration points for all meshes.

\section{Acknowledgment}

The first author would like to thank the Coordination of Improvement of Higher Education Personnel(CAPES), Brazil and State Univesity of Campinas (UNICAMP), Brazil, for 
financial support for this work.

\section{References}

[1] S. Timoshenko and J. M. Gere. Theory of Elastic Stability. McGraw-Hill, New York, second edition, 1961.

[2] P. Sollero. Fracture mechanics analysis of anisotropic laminates by the boundary element method. PhD thesis, Wessex Institute of Technology, 1994.

[3] E. L. Albuquerque. Numerical analysis of dynamic anisotropic problems using the boundary element method. PhD thesis, Unicamp, Dept. Mec. Comput., July 2001. In Portuguese.

[4] W. P. Paiva. Análise de problemas estáticos e dinâmicos em placas anisotrópicas usando o método dos elementos de contorno. PhD thesis, Universidade Estadual de Campinas, Campinas, 2005. In Portuguese.

[5] E. L. Albuquerque, P. Sollero, W. Venturini, and M. H. Aliabadi. Boundary element analysis of anisotropic kirchhoff plates. International Journal of Solids and Structures, 43:4029-4046, 2006.

[6] A. Reis et al. Computation of moments and stresses in laminated composite plates by the boundary element method. Engineering Analysis with Boundary Elements, 35:105-113, 2011.

[7] P. C. M. Doval, E. L. Albuquerque, and P. Sollero. Stability analysis of composite laminate plates under non-uniform stress filds by the boundary element method. In XI International Conference on Boundary Element and Meshless Techniques, july 2011.

[8] X. Gao. The radial integration method for evaluation of domain integrals with boundary only discretization. Engn. Analysis with Boundary Elements, 26:905-916, 2002.

[9] L. J. M. Jesus, E. L. Albuquerque, K. R. Sousa, and P. Sollero. Further developments in the radial integration method. In XXXI CILAMCE - Congresso Ibero Latino Americano de Mtodos Computacionais em Engenharia, Buenos Aires, Argentina, November 2010.

[10] M. H. Aliabadi. Boundary element method, the application in solids and structures. John Wiley and Sons Ltd, New York, 2002.

[11] P. Sollero and M. H. Aliabadi. Fracture mechanics analysis of anisotropic plates by the boundary element method. Int. J. of Fracture, 64:269-284, 1993.

[12] E. L. Albuquerque, P. Sollero, and W. P. Paiva. The radial integration method applied to dynamic problems of anisotropic plates. Communications in Numerical Methods in Engineering, 23:805-818, 2007. 
[13] E. L. Albuquerque and M. H. Aliabadi. A boundary element formulation for boundary only analysis of thin shallow shells. CMES - Computer Modeling in Engineering and Sciences, 29:63-73, 2008.

[14] P. W. Partridge. Towards criteria for selection approximation functions in the dual reciprocity method. Engineering Analysis with Boundary Elements, 24:519-529, 2000.

[15] M. A. Golberg, C. S. Chen, and H. Bowman. Some recent results and proposals for the use of radial basis functions in the bem. Engineering Analysis with Boundary Element, 23:285-296, 1999. 